and Functional Polymers

Manuscript Number: REACT-D-16-00650R1

Title: The Influence of Adding Functionality to Dispersant and Particle Core Compositions in Non-Aqueous Dispersion Polymerization

Article Type: Research Paper

Keywords: Macromer dispersant; Hydroxyl functionality; Non-aqueous dispersion; Functionalized copolymers; Solubility parameters

Corresponding Author: Dr. Robin A. Hutchinson, PhD

Corresponding Author's Institution: Queen's University

First Author: Weiwei Yang

Order of Authors: Weiwei Yang; Robin A. Hutchinson, PhD 


\title{
The Influence of Adding Functionality to Dispersant and Particle Core Compositions in Non-Aqueous Dispersion Polymerization
}

\author{
Weiwei Yang ${ }^{\mathrm{a}, \mathrm{b}}$ and Robin A. Hutchinson ${ }^{\mathrm{a}, 凶}$ \\ ${ }^{a}$ Department of Chemical Engineering, Dupuis Hall, Queen's University, Kingston, \\ Ontario,K7L 3N6, Canada (robin.hutchinson@queensu.ca) \\ Phone: +1 613-533-3097 Fax: +1 613-533-6637 \\ ${ }^{\mathrm{b}}$ Currently at School of Textile and Clothing, Nantong University, Nantong, Jiangsu Province, \\ 226019, People's Republic of China
}

\begin{abstract}
Nano-sized polyacrylate colloids $(<200 \mathrm{~nm})$ at high solids level $(\sim 60 \mathrm{wt} \%)$ are prepared via non-aqueous dispersion (NAD) semibatch polymerization using $n$-butyl methacrylate (BMA)based macromer dispersants of varying chain lengths and levels of functionality provided by incorporation of 2-hydroxyethyl methacrylate (HEMA) comonomer. The effect of building the complexity of the core polymer recipe from methyl acrylate homopolymer to a five-monomer composition is systematically studied. While average particle size increased with the introduction of functional comonomers, the resulting NAD systems remained stable for all core compositions using both BMA macromers and a copolymer macromer containing 5 mol\% HEMA. However, introduction of the HEMA functionality to the dispersant at levels of $>5 \mathrm{~mol} \%$ led to destabilization of the dispersion. The polarity differences between the core compositions and dispersant polymers and the mixed continuous medium, as represented by Hansen solubility parameters, are qualitatively used to interpret the nucleation of particles and the effectiveness of the dispersants.
\end{abstract}

\section{Keywords}

Macromer dispersant; Hydroxyl functionality; Non-aqueous dispersion; Functionalized copolymers; Solubility parameters 

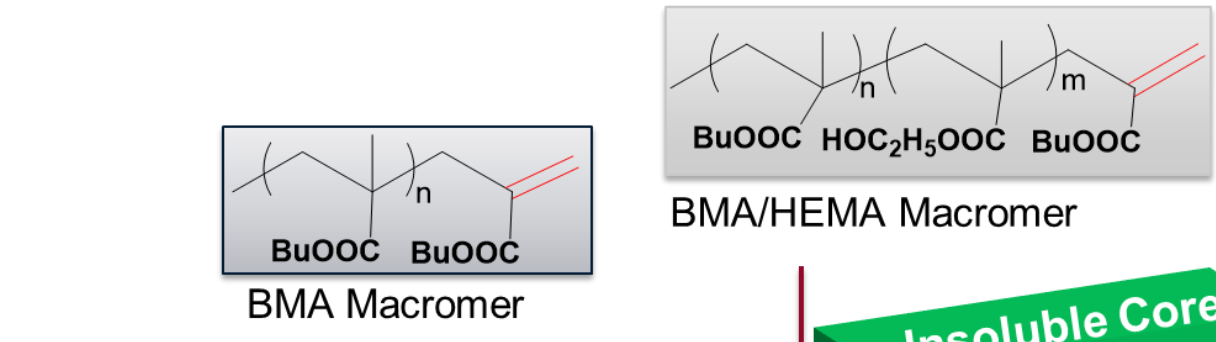

BMA/HEMA Macromer

BMA Macromer

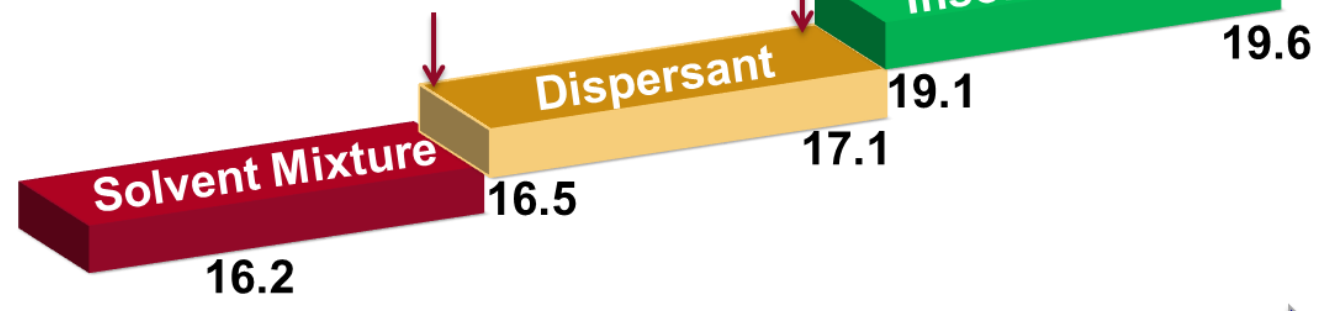

\section{Increased Polarity}




\section{Introduction}

Non-aqueous dispersion (NAD) polymerization has been employed by the automotive coatings industry to prepare nano-sized colloids with high solids content (> $50 \mathrm{wt} \%$ ) and low viscosity for decades.[1],[2] The utilization of these components in hybrid polymer acrylic coating systems continues to expand, as they allow the reduction of solvent level while lowering energy consumption during the curing stage. The dispersion is produced in a batch or semibatch system that starts as a homogeneous phase in which monomer, solvent, and polymeric dispersant (stabilizer) are well mixed. Upon the addition of initiator, newly generated polymer chains exceeding a critical length precipitate from the continuous phase due to their poor solubility and form precursor particles that coalesce in the presence of dispersant to form stable nanoparticles. Particle growth proceeds by the adsorption or absorption of initiator, monomers, dispersant, polymeric radicals and dead polymer chains, with polymerization occurring predominantly in the particle phase.[2]

Reactive dispersants with vinyl unsaturation are effective steric stabilizers that prevent colloidal flocculation in dispersion systems, although unreactive dispersant homopolymers[3] and block[4] or random copolymers[5] are also employed. Various vinyl-terminated macromer (macromonomer) dispersants such as poly(2-alkyl-2-oxazoline),[6] poly(ethyl glycol),[7] and poly(dimethyl siloxane) (PDMS)[8] have been used to produce poly(methyl methacrylate) (PMMA) and polystyrene (PST) particles either in polar (alcohol/water) or non-polar (alkyl hydrocarbons) solvents. These dispersion systems studied usually have less than $20 \mathrm{wt} \%$ dispersant relative to monomer in order to produce micron-size particles with low solids content $(<40 \mathrm{wt} \%)$. However, higher dispersant levels $(\sim 50 \%$ relative to monomer) are generally applied in the industrial process to produce dispersions with high solids content (> 50 wt $\%$ ) and nanosized particles.[1],[2]

Our previous work[9] showed that a poly(butyl methacrylate) macromer dispersant was more efficient than a vinyl poly(butyl methacrylate-co-methacrylic acid-glycidyl methacrylate) grafted dispersant. The latter was produced by attaching the vinyl functionality of glycidyl methacrylate (GMA) by reaction of the epoxy group with the carboxyl group of methacrylic acid (MAA) groups randomly incorporated in a copolymer consisting of predominantly butyl methacrylate (BMA) units, following a procedure reported in the patent literature.[1] Although 
the two dispersants were of similar chain length (number average molar mass $\left(M_{n}\right)$ of 5000 $6000 \mathrm{Da}$ ) and had similar vinyl content, the macromer dispersant was incorporated into the methacrylate/acrylate copolymer NAD product at a higher level, leading to a reduction of particle size.[9] The lowered efficiency of the grafted dispersant can be attributed to the stochastic distribution of the vinyl functionality among the polymer chains, as demonstrated by comparing the molar mass distribution (MMD) of the soluble polymer recovered from the dispersion to that of the initial dispersant, combined with NMR analysis: the shorter chains were unfunctionalized and ineffective as dispersant, while the chains of longer length containing multiple vinyl groups became attached to the particles. This uneven distribution of functionality was in accord with simulations of the radical polymerization process used to synthesize the grafted dispersant.[10] In contrast, MMD of the soluble polymer recovered from the dispersion produced with macromer exactly overlayed that of the initial dispersant material: with exactly one terminal double bond per chain, all macromer chains are equally likely to react, independent of chain length.[9]

While the utilization of macromer as dispersant improved the NAD system (decreased particle size, with a greater fraction of the dispersant attached or adsorbed to the particles), its level of incorporation was found to be dependent on the composition of the core polymer being produced. Specifically, homopolymer particles produced using methyl acrylate (MA) were significantly smaller than copolymer nanoparticles of MA with methyl methacrylate (MMA) due to the difference in reactivity of a macromer terminal vinyl group with methacrylate and acrylate radicals[9]; while the macromer terminal double bond readily adds to acrylate radicals, it predominantly undergoes a $\beta$-scission chain transfer reaction with methacrylate radicals.[11] Thus, the incorporation of the macromer into the NAD particles was found to be higher for the MA homopolymer than for the MA/MMA copolymer. However, even for the most favorable result obtained with macromer dispersant and MA core, $\sim 40 \%$ of the added dispersant remains in the continuous phase at the end of reaction.[9]

In addition to the reactivity of the dispersant, the influence of core compositions on NAD properties is also of interest. Only a limited number of studies have focused on copolymer colloidal systems, with most using dispersants containing labile hydrogen atoms. For example, styrene (ST)-based copolymer dispersion stabilized by poly(vinyl pyrrolidone) (PVP) in ethanol/water was produced with butyl acrylate (BA) and 2-ethylhexyl acrylate (EHA);[12] it 
was found that particle size increased with a higher acrylate content in the copolymer, but not with the choice of BA vs EHA. A comparable result was reported for ST copolymerized with methacrylates (GMA, MMA, and ethyl methacrylate) using a similar solvent and stabilizer.[13] Song et al.[14] prepared micron-sized poly(ST-co-acrylic acid) particles with PVP via a twostage method in which the addition of acrylic acid to the system was delayed to prevent secondary nucleation. The two-stage experimental procedure was also applied to make narrower distributed poly(ST/dye-monomer) colloids, as a broader size distribution was seen in the comparable one-stage batch study.[15] When BA was reacted with MMA in a methanol/water blend stabilized by PVP, the particle size of the core-shell polymer increased with the increased fraction of MMA, and a PMMA-rich core structure was formed due to the higher incorporation of MMA to the copolymer.[16]

There are significant differences between these previous studies and the NAD formulations used in the coatings industry, which are high-solids dispersions of a more polar core acrylic (co)polymer in a less polar solvent mixture stabilized by a reactive dispersant. The composition of the NAD acrylic colloids is often varied to ensure compatibility with other components, to introduce functional groups, and to tailor physical properties (e.g., glass transition temperature, curing temperature and haziness) of a particular coatings formulation.[1],[2],[5] Thus, it is important to study the influence of core composition on the performance of the macromer dispersants being investigated. In addition, the impact of adding

hydroxyl groups to the BMA methacrylate macromer is examined, as dispersant functionality is required for commercial NAD applications to reduce the impact of low MW non-functionalized chains in the final coatings formulation.[5] Hansen solubility parameters are introduced as a means to interpret the effect of the dispersant composition on the NAD stability and average particle size.

\section{Experimental Section}

\subsection{Materials}

The monomers, methyl acrylate (Sigma-Aldrich, 99\%), styrene (Sigma-Aldrich, $\geq 99 \%$ ), 2-hydroxyethyl acrylate (HEA, Sigma-Aldrich, 96\%), methacrylic acid (MAA, Sigma-Aldrich, 99\%) and glycidyl methacrylate (GMA, Sigma-Aldrich, 97\%), were used as received. The 
initiator, 2,2'-azobis-(2-methylbutyronitrile) (Vazo ${ }^{\circledR}$ 67, E. I. du Pont), was utilized as received. Solvents used in the study, also without purification, were xylene (Sigma-Aldrich, $\geq 98.5 \%$ ), pxylene (Sigma-Aldrich, $\geq 99 \%$ ), deuterochloroform $\left(\mathrm{CDCl}_{3}\right.$, Sigma-Aldrich, 99.8 atom\% D), heptane (Sigma-Aldrich, $\geq 99 \%$ ), and butyl acetate (Sigma-Aldrich, $\geq 99 \%$ ).

The BMA-based macromer dispersants were kindly supplied by Axalta Coating Systems as $60 \mathrm{wt} \%$ polymer solution in xylene, synthesized through cobalt chain transfer polymerization.[17] The number-average $\left(M_{n}\right)$ and weight-average $\left(M_{w}\right)$ molar masses of the dispersant polymers are summarized in Table 1, as measured by size-exclusion chromatography (SEC) and checked via proton nuclear magnetic resonance $\left({ }^{1} \mathrm{H}\right.$ NMR) (see Supplementary Data), the same means utilized in our previous work.[9] Two BMA homopolymer macromers of differing length, M1 and M2, were used during the systematic variation of core composition. A second series of macromers were synthesized to study the influence of dispersant composition on NAD synthesis; these are also BMA-based with HEMA comonomer level varied from $0\left(\mathrm{BH}_{0}\right.$, BMA homo-macromer) to $15\left(\mathrm{BH}_{15}\right) \mathrm{mol} \%$, with MWs also summarized in Table 1 . The influence of core compositions on NAD properties was also investigated using $\mathrm{BH}_{5}$, the macromer dispersant containing 5 mol\% HEMA.

\subsection{Non-Aqueous Dispersion Polymerization}

A 0.6 L glass reactor was utilized to conduct all semibatch experiments, with the operating procedure, feeding schedule, ratio of dispersant, monomers, solvents and initiator kept the same as described in our previous publication, as was the synthetic procedure.[9] Two thirds of the dispersant solution and heptane were initially loaded to the reactor and heated to $92{ }^{\circ} \mathrm{C}$, a temperature slightly below the normal boiling point of heptane. An initiator shot of Vazo 67 dissolved in n-butyl acetate was added, followed by the addition of monomers, the remainder of the dispersant solution and Vazo 67 dosed at a constant flow rate in $210 \mathrm{~min}$. The semibatch feeding of monomer keeps the instantaneous monomer conversion in the reactor high (> 95\%),[9] ensuring that composition of the copolymer produced remains uniform. The reaction mixture was then held in batch mode for 45 min before an initiator solution of Vazo 67 and butyl acetate was pumped to the reactor for $30 \mathrm{~min}$ and held for an additional $60 \mathrm{~min}$. While the total mass of monomer was kept constant for all experiments, the compositions were systematically 
varied from the acrylate homopolymer recipe to five-monomer core, as specified by Table 2 . The reproducibility of dispersion polymerization was checked with dispersant M1 and the MA homopolymer core, as documented in the Supplementary Data.

\subsection{Characterization}

The polymer remaining in the continuous phase was quantified after destabilizing the final dispersion to separate out the particles using a procedure described previously.[9] A combination of gravimetry and ${ }^{1} \mathrm{H}$ NMR was used to determine the amount of the added dispersant that remained in solution and thus calculate $w_{\text {inc }}$, the fraction of dispersant attached or adsorbed to the particle phase. Spectra were measured using a Bruker Avance-400 $\mathrm{MHz}$ spectrometer on dried polymer dissolved in $\mathrm{CDCl}_{3}$, with details described in the previous publication.[9] The value of $w_{\text {inc }}$ did not vary with repeated washings, indicating that it is a reasonable indication of dispersant fraction covalently attached to the nanoparticles.

Polymer MW averages and molar mass distributions (MMDs) were measured for all dispersants, for the soluble polymer recovered from the NADs, and for the final NAD products. The SEC analysis of lower MW samples (dispersant and soluble polymer) was done using a Waters 410 differential refractometer (DRI) operated at $35^{\circ} \mathrm{C}$, with THF as eluent at a flow rate of $0.3 \mathrm{~mL} \cdot \mathrm{min}^{-1}$. The DRI detector was calibrated by 10 narrow PST standards with MW from 870 to $355000 \mathrm{Da}$. The MMDs for the NAD polymer (higher MW samples) were analyzed by SEC using a Viscotek 270 max separation module with triple detectors. THF was used an eluent at $1.0 \mathrm{~mL} \cdot \mathrm{min}^{-1}$ at $40{ }^{\circ} \mathrm{C}$. The reported values are based upon PST calibration, without any correction.

Average particle diameter and size distribution were measured on a Malvern Zeta-Sizer Nano ZS using dynamic light scattering (DLS) with an angle of $173^{\circ}$ at $25^{\circ} \mathrm{C}$ in a quartz cuvette. NAD samples were diluted using a solvent mixture (50 wt \% heptane and $50 \mathrm{wt} \% \mathrm{p}$-xylene), with the refractive index $(1.418)$ and viscosity $(0.562 \mathrm{cP})$ of the binary solvent mixture calculated via the Lorentz-Lorenz formula[18] and the Grunberg and Nissan equation,[19] respectively. The refractive index of polymer is 1.59 , as provided by Axalta Coating Systems. Unfortunately, the particles could not be examined using microscopy due to the low glass transition temperature of the core formulations. 


\section{Results and Discussion}

\subsection{Introducing Functionality to the MA Based Core}

In our previous work, we demonstrated that a poly(butyl methacrylate) macromer, with its uniform distribution of terminal vinyl groups, is a more effective dispersant for production of acrylic nanoparticles by non-aqueous dispersion polymerization than a BMA-based dispersant of similar MW with the vinyl functionality distributed randomly among the chains.[9] The conclusion was based upon synthesis of MA homopolymer and MMA/MA copolymer of NAD particles. However, commercial NAD formulations are often crosslinked in situ by adding epoxy and carboxyl functionality with GMA and MAA, with HEA added to introduce reactive hydroxyl groups for post-production curing, and ST and alkyl (meth)acrylates added to adjust the refractive index and glass transition temperature.[1] Thus, it is necessary to demonstrate that these more complex recipes are also well-stabilized by the macromer dispersant. Two BMA macromers of different chain length (and thus different concentration of vinyl groups for the same mass) were chosen for comparison.

The dispersant solution, consisting of $60 \mathrm{wt} \%$ polymer in xylene solution, is added as a reactive component in the starved-feed semibatch NAD process to stabilize the nanoparticles formed by precipitation of the polar acrylic polymer produced via radical polymerization of the monomer mixture co-fed with initiator. As described more fully elsewhere,[9] the formation of nanoparticles is first visually observed $\sim 1 \mathrm{~h}$ after the start of polymerization, with solids content increasing over the course of reaction to yield the final NAD mixture containing $60 \mathrm{wt} \%$ polymer in xylene/heptane solvent. It is only after the formation of particles that higher-MW polymer is formed, as shown by the MMDs in Figure 1 for the MA/ST copolymerization (recipe "AS" in Table 2) produced with dispersant M1. The polymer formed before nucleation is of lower MW as it is generated by polymerization in the continuous phase; after nucleation much of the reaction occurs in the particle phase, leading to higher polymerization rate and production of much higher MW polymer.[20],[21] The final mixture has a bimodal MMD, with the low-MW mode overlapping the MMD of the original dispersant, an indication of the presence of unreacted macromer. 


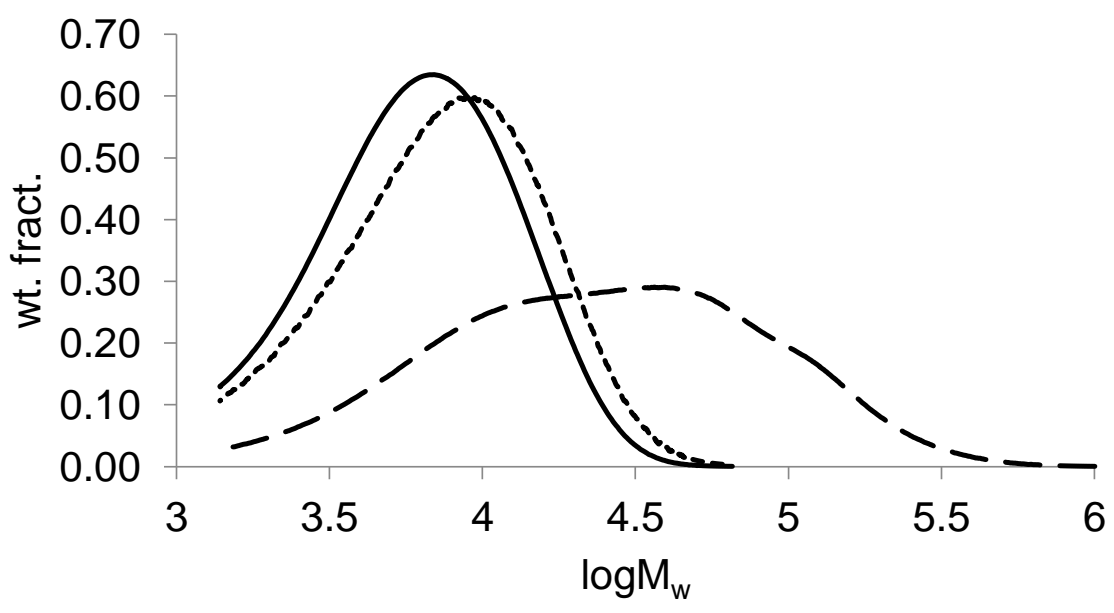

Figure 1. MMDs of Dispersant M1 (solid line), a dispersion sample taken 60 min after the start of polymerization (dotted line), and the final dispersion product (dashed line) from recipe AS (see Table 2).

The poly(methyl acrylate) (PMA) NAD produced with M1 has a $\mathrm{M}_{\mathrm{w}}$ about $80000 \mathrm{Da}$, while that produced with dispersant M2 is just over $63000 \mathrm{Da}$. The difference is due to the higher concentration of reactive double bonds for the lower MW M1 dispersant. An opposite relationship between NAD and macromer $\mathrm{M}_{\mathrm{w}}$ values was observed during the production of MMA/MA copolymer, with the lower MW macromer leading to lower MW final product; in addition, the $\mathrm{M}_{\mathrm{w}}$ values of the copolymer were significantly lower than those of MA homopolymer synthesized under identical conditions.[9] These results were attributed to the chain-transfer reaction that occurs when a methacrylate radical encounters the methacrylate macromer double bond: rather than incorporating into the growing chain (as occurs when reacting with an acrylate radical), a $\beta$-scission (chain-transfer) reaction can happen, hence reducing polymer MW.[11]

The effect of adding styrene to the MA system is shown in Figure 2. $M_{w}$ values obtained for NADs made with the MA-only core are consistently higher than those of NADs made with MA/ST copolymer. (All other NAD compositions studied led to insoluble crosslinked particles.) The relative rate of addition and $\beta$-scission when macromer adds to a styrenic radical is not wellstudied. As reported by Cacioli et al.,[11] the MW of graft copolymer formed with methacrylate macromer and ST was significant lower than that of ST homopolymer produced at identical reaction conditions, a result likely due to the formation of sterically hindered radicals which may 
be less reactive for further monomer addition. It is also known that acrylate polymer MWs are reduced when ST is added to a homogeneous polymerization system due to the slower polymerization rate.[22] Both explanations, lowered propagation rates and chain transfer of the styrenic radicals to the macromer, can be applied to explain the lower $\mathrm{M}_{\mathrm{w}}$ seen for the MA/ST core. While an interesting kinetic question, the important finding is that the $\mathrm{M}_{\mathrm{w}}$ values of the NAD are not greatly affected by the addition of ST to the recipe, remaining greater than 55000 Da.

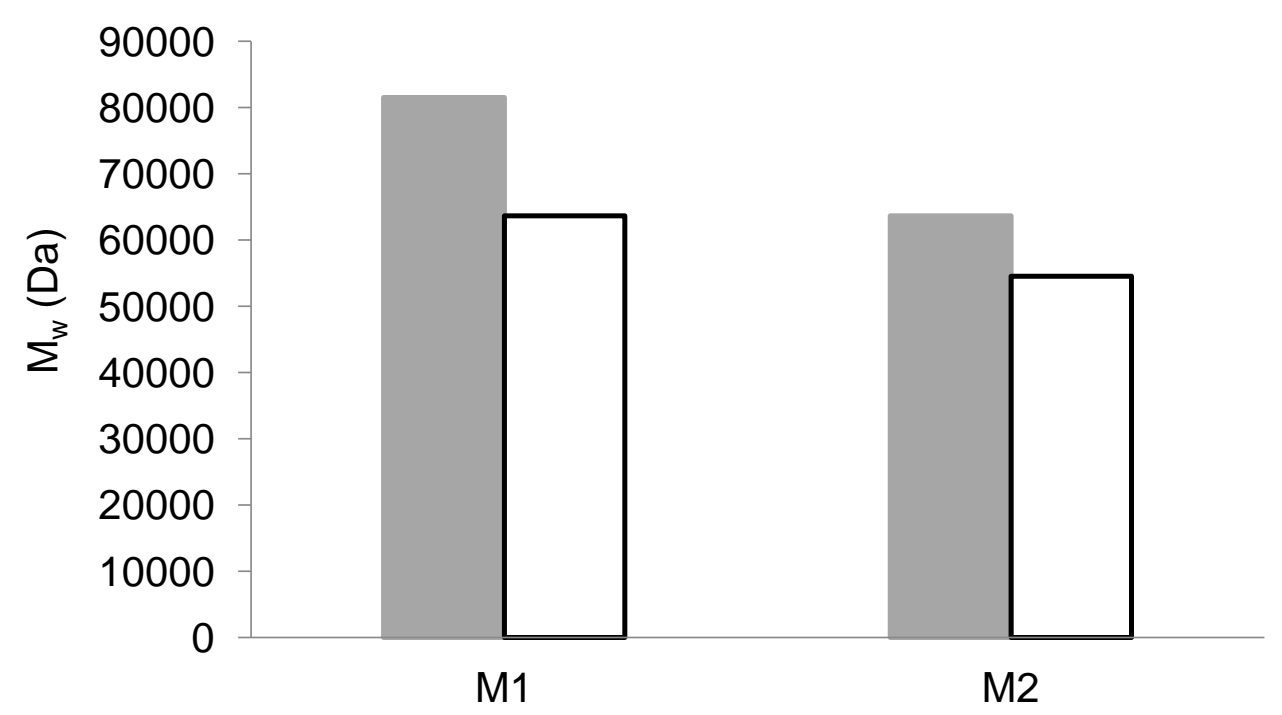

Figure 2. Weight-average molar mass of MA homopolymer (filled bars, sample named as "A" in Table 2) and MA/ST copolymer (unfilled bars, sample named as "AS" in Table 2) NADs prepared using two different BMA macromers.

The influence of varying the core compositions on the incorporated fraction of macromer dispersant $\left(w_{\text {inc }}\right)$ is shown in Figure 3; for this discussion all results, including NADs with crosslinked cores, are considered. As described in the experimental section, $w_{\text {inc }}$ is calculated by measuring the amount of soluble polymer that remains in the continuous phase; thus a higher value indicates a greater fraction of the dispersant is attached to the core polymer to form a stabilizing graft. High utilization of the dispersant is desired to minimize material usage, a factor favoring the use of macromer over dispersants of similar MW with the vinyl functionality 
distributed randomly among the chains.[9] The $w_{\text {inc }}$ value of 0.5-0.6 measured for MA homopolymer does not vary greatly when crosslinking (AX) and hydroxyl (AHX) functionality is added to the recipe, but is slightly lowered with the addition of ST (compare AS vs A, and AHSX vs AHX results in Figure 3). The cause of this decrease is not obvious: as mentioned previously, it may be that ST radicals preferentially undergo a chain transfer rather than an addition reaction when encountering the vinyl functionality at the macromer chain end. In general, the lower MW macromer M1 is more effective than M2, as a higher vinyl concentration results from the same added mass of macromer to the NAD systems.

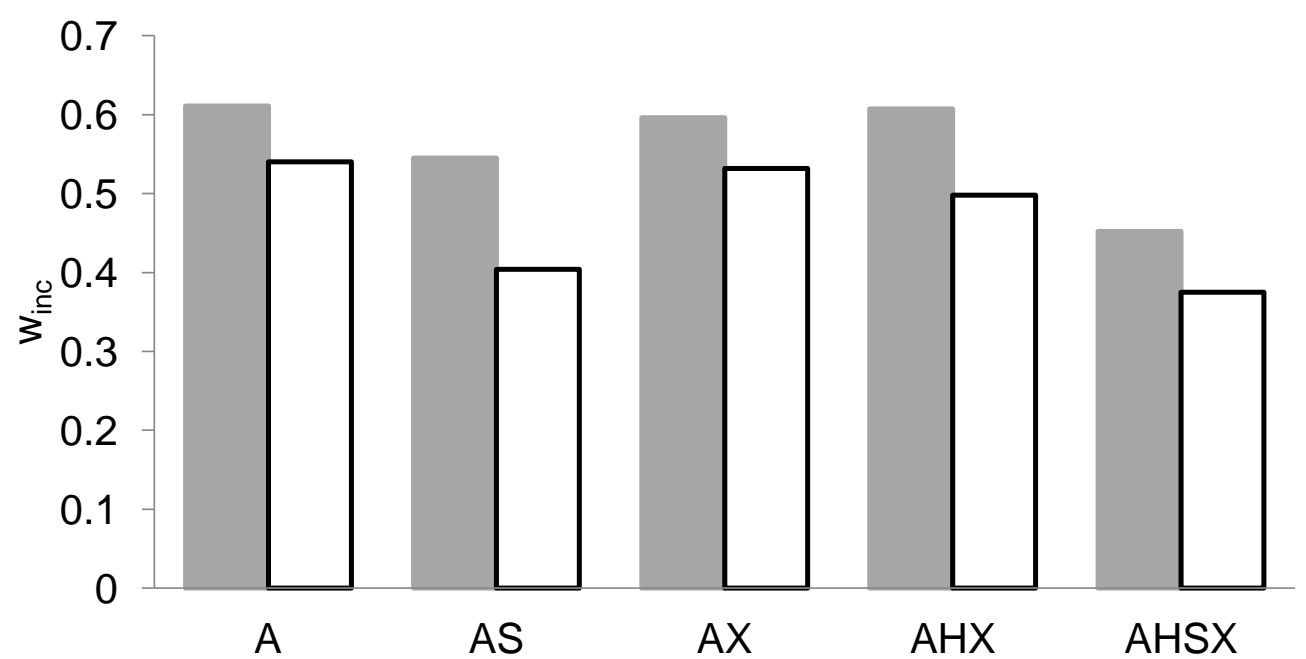

Figure 3. The mass fraction of incorporated dispersant $\left(w_{\text {inc }}\right)$ measured for NADs made with two macromer dispersants M1 (filled bars) and M2 (unfilled bars) and various core compositions (see Table 2).

Figure 4 shows that average particle size $\left(d_{p}\right)$ for all NAD compositions remains less than $180 \mathrm{~nm}$, with smaller particles produced using the lower MW dispersant (M1) that is also more effectively incorporated (Figure 3) due to the higher concentration of double bond in the M1 system. An increase in particle size is caused by the addition of ST to the recipe, as seen by comparing A to AS and AHX to AHSX. Once again, this change correlates with a corresponding decrease in the values of $w_{\text {inc }}$. However, addition of crosslinking and hydroxyl groups to the recipe also leads to an increase in particle size (AX and AHX vs A), although the value for $w_{\text {inc }}$ 
did not significantly change. These monomers (GMA, MAA and HEA) introduce additional polarity to the core, as discussed in the followed section.

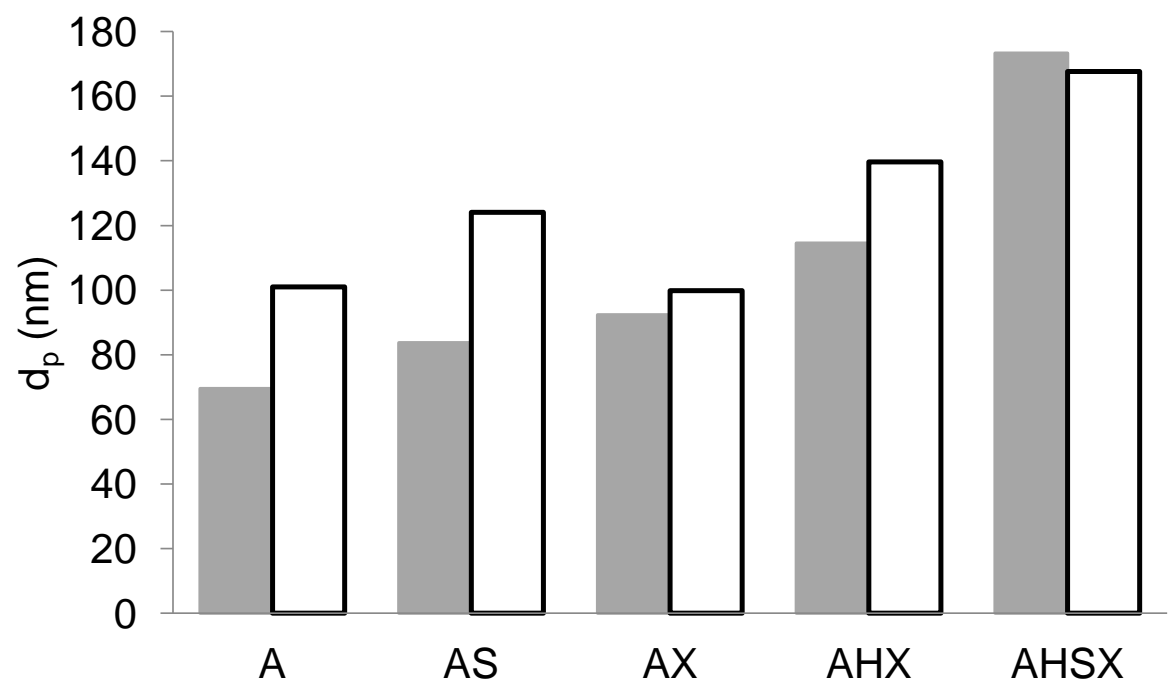

Figure 4. Average particle size measured for NADs made with two macromer dispersants M1 (filled bars) and M2 (unfilled bars) and various core compositions (see Table 2).

Although the reason behind the increased particle size in the presence of functional monomers is not clear, an important general conclusion can be drawn from this set of studies. The BMA macromers under investigation are able to stabilize poly(acrylic) nanoparticle $\left(d_{p}<200 \mathrm{~nm}\right)$ dispersions of $60 \mathrm{wt} \%$ solids content that incorporate the range of monomers typically employed to tailor end-use properties of the final coatings formulation. Even for the most complex case (AHSX), usage of the macromer results in particle sizes comparable to a MMA/MA (non-functional core) NAD produced using a randomly grafted dispersant,[9] and the utilization of the dispersant remains higher.

\subsection{Introducing Hydroxyl Functionality to the Macromer Dispersant}

The BMA macromer has been demonstrated to be effective at stabilizing the NAD particles with average size less than $200 \mathrm{~nm}$. However, while incorporated with the core particle at levels higher than the randomly functionalized stabilizer, roughly half of the dispersant 
remains in the continuous phase in the final dispersion. It is for this reason that dispersants usually contain hydroxyl groups,[1] so that the chains remaining in solution can undergo crosslinking during the curing process of the coatings; [23] higher hydroxyl levels speed up the curing procedure and improve damage resistance.[24] Thus, BMA/HEMA macromers with varied HEMA levels were synthesized and tested for their effectiveness as dispersants in this study.

The BMA/HEMA macromers with higher HEMA levels (10\% and $15 \%$ in the copolymer composition, labelled as $\mathrm{BH}_{10}$ and $\mathrm{BH}_{15}$ in Table 1) were first investigated for stabilization of MA homopolymer nanoparticles. Surprisingly, it was found that the products synthesized with these macromers were unstable, with flocculation of the particles observed immediately after the reaction, as depicted in Figure 5. As discussed in more detail later, we hypothesize that the higher polarity of the HEMA-containing macromers favors their partition to the more polar particle phase rather than the less polar continuous phase, causing their ineffectiveness as stabilizers: rather than extending into the non-polar medium or attaching on the particle surface, the BMA/HEMA macromer may be buried inside of the particles.

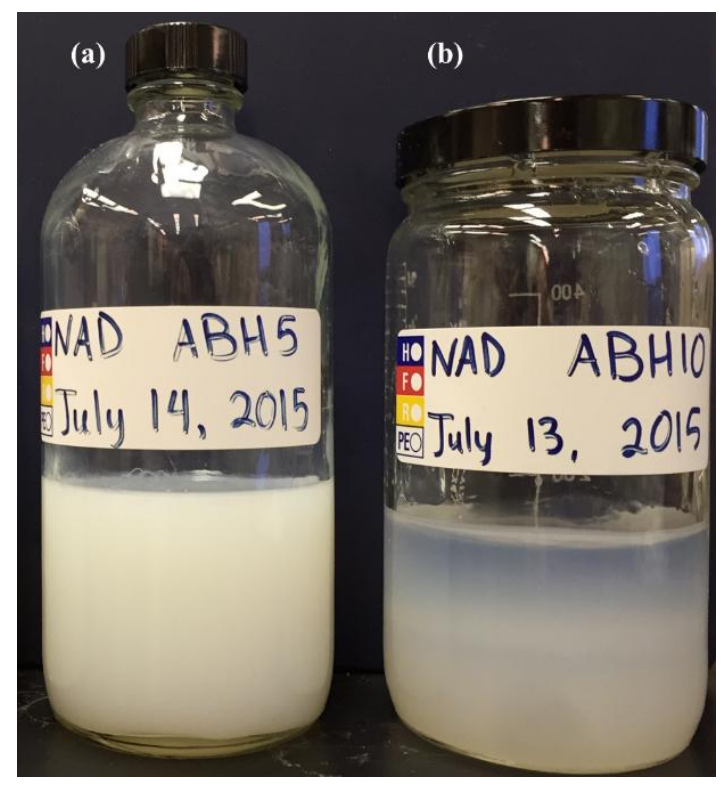

Figure 5. Non-aqueous dispersions of MA homopolymer core prepared with two different HEMA-containing macromers: (a) stable NAD produced with $\mathrm{BH}_{5}$ (5 mol\% HEMA); (b) unstable NAD produced with $\mathrm{BH}_{10}(10$ mol\% HEMA). 
With the lowest HEMA content macromer $\mathrm{BH}_{5}$, however, stable product (no coagulation of particles observed weeks after the reaction completed) was formed over the entire range of compositions studied, with $d_{p}$ increasing from 90 to $150 \mathrm{~nm}$ as the recipe complexity increased from homopolymer to the most complex copolymer (AHSX, see Table 2).[25] As shown by Figure 6, the MMD of the final MA homopolymer dispersion produced using the HEMAcontaining $\mathrm{BH}_{5}$ macromer is very similar to that produced with the BMA macromer, as is its relationship to the MMD of the original dispersant. However, a significant increase in the fraction of incorporated dispersant $\left(w_{i n c}>0.9\right)$ was measured for $\mathrm{NAD} \mathrm{A-BH}{ }_{5}$ compared to the values of 0.5-0.6 found for $\mathrm{NAD} \mathrm{A-BH}$. While this might indicate increased effectiveness of the dispersant, the average particle size produced was actually slightly greater (92 vs $83 \mathrm{~nm}$ ) using the HEMA-containing macromer. The finding is more likely an artifact of the experimental procedure used to separate the particles from the continuous phase, facilitated by the addition of heptane. The MMDs of the original macromer dispersants and the soluble polymers recovered from the extraction by heptane are shown in Figure 7. The MMD of the BMA macromer and the corresponding soluble polymer recovered from the NAD produced are almost identical, a result shown before[9] and attributed to the even distribution of vinyl group among the BMA macromer chains. However, the soluble polymer recovered from the NAD produced with dispersant $\mathrm{BH}_{5}$ is of lower $\mathrm{MW}$ than the original macromer. While the desired role of macromer is to react with the core chains and stabilize onto the surface of the particle, it may also physically absorb (or react) into the interior of the particles, with higher $\mathrm{MW} \mathrm{BH}_{5}$ dispersant chains containing more HEMA units preferentially attaching to the nanoparticles produced. In addition, the higher polarity of $\mathrm{BH}_{5}$ and its poorer solubility in heptane (as compared to BMA macromer) may cause the higher MW dispersant chains to adsorb to the particle surface after addition of the extracting solvent heptane, such that the chains of shorter length (and perhaps reduced HEMA content) are preferentially recovered from the dispersion during post-reaction analysis. This possibility was checked by using an $80 / 20(\mathrm{w} / \mathrm{w})$ heptane/xylene mixture to separate the particles from the continuous phase. This procedure reduced the value of $w_{\text {inc }}$ to 0.60.7 for the series of NAD materials produced with $\mathrm{BH}_{5}$, and also shifted the MMD of the soluble polymer recovered from the NAD closer to that of the original dispersant, as seen in Figure 7c. From this analysis, it can be inferred that while the increased polarity of the HEMA-containing 
dispersant causes it to partition to the particles at increased levels compared to the homopolymer dispersant, the level of covalent bonding does not necessarily also increase.

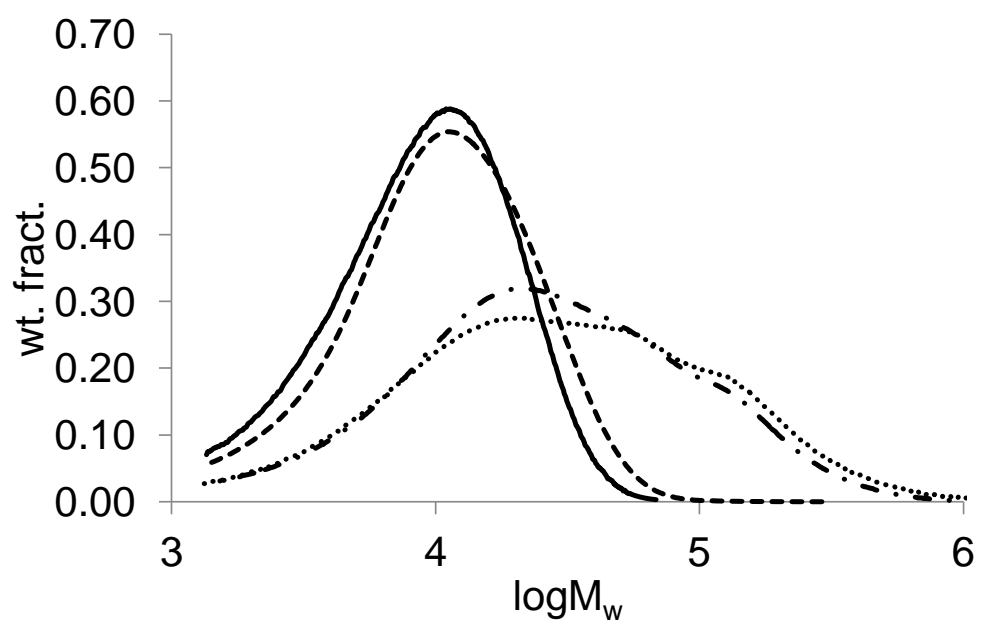

Figure 6. MMDs of macromer dispersants and $\mathrm{NADs}$ : $\mathrm{BH}_{0}$ (solid line), $\mathrm{BH}_{5}$ (dashed line), $\mathrm{NAD}$ $\mathrm{A}-\mathrm{BH}_{0}$ (dotted line) and $\mathrm{NAD} \mathrm{A}-\mathrm{BH}_{5}$ (dashed-dotted line).
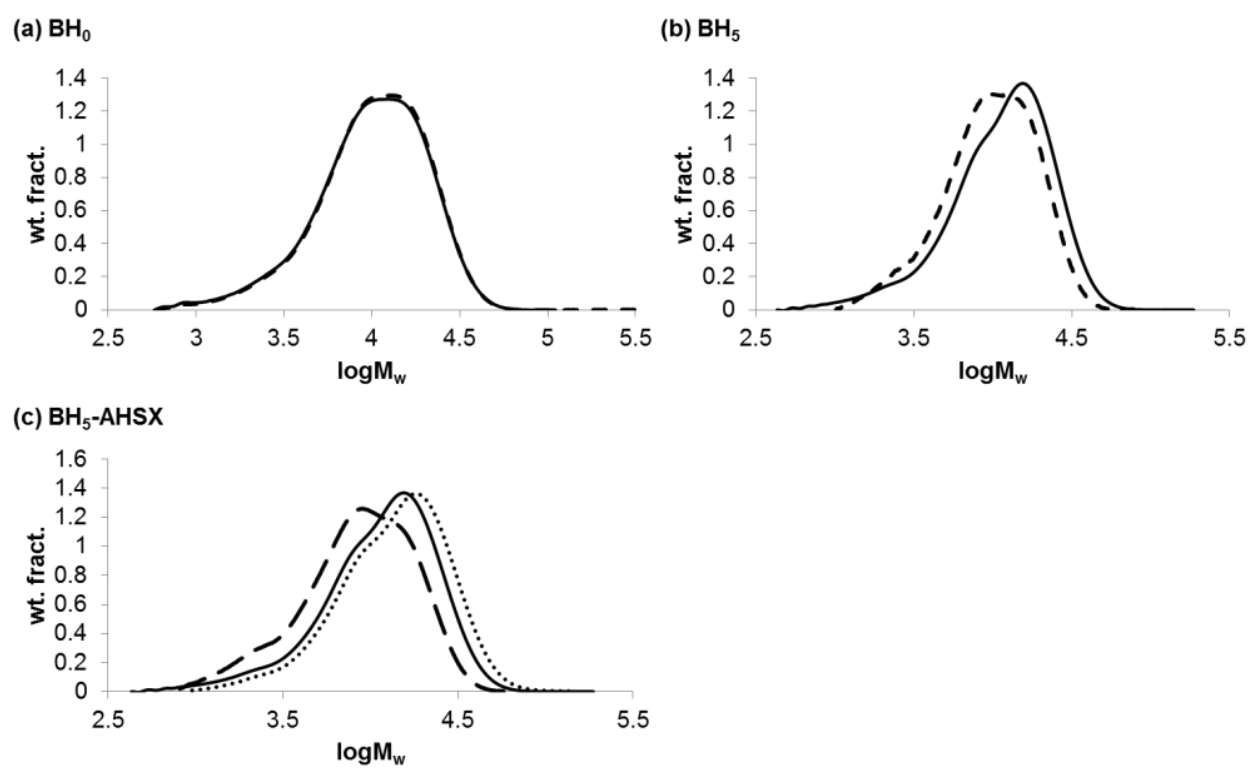

Figure 7. A comparison of the dispersant MMDs (solid lines) with the soluble polymer recovered from the final non-aqueous dispersion by heptane treatment (dashed lines): (a) $\mathrm{BH}_{0}$ and NAD A$\mathrm{BH}_{0}$; (b) $\mathrm{BH}_{5}$ and NAD A-BH ; (c) $\mathrm{BH}_{5}$ and NAD AHSX-BH 5 . In (c), the MMD of the soluble polymer exacted by $80 / 20$ heptane/xylene mixture is also shown (dotted line). 
While stable non-aqueous dispersions using the complete range of compositions were successfully produced using the HEMA-containing dispersant $\mathrm{BH}_{5}$, there are strong indications that the system is approaching the limits of stability: the use of the dispersant with higher HEMA content $\left(\mathrm{BH}_{10}\right)$ resulted in an unstable system, and the partition behavior of the soluble polymer was significantly different during the separation of the particles and continuous phase of the NAD product. The interpretation of this behavior can be aided by the consideration of the relative compatibility of the solvent mixture, dispersant, and insoluble core particle, using Hansen solubility parameters.

Solubility parameters are often applied in the coatings industry to ensure compatibility between solvent and polymer in a homogeneous mixture. Here, the concept is applied to the heterogeneous NAD system, with the goal of understanding the performance of BMA/HEMA macromers in particular. It is desirable that the core polymer is incompatible with the solvent mixture to promote particle nucleation; while the dispersant should be compatible with the solvent such that after it reacts with the core polymer chains, it can anchor on the particle surface and extend into solvent to stabilize the particles.

A few literature studies have employed solubility parameters to interpret batch dispersion systems with varied monomer[26] and solvent compositions.[27],[28] In one effort, dispersion polymerization of five different alkyl methacrylate monomers was investigated in a methanol/water mixture (polar medium) with stabilizer PVP; the solubility parameter decreased for the longer alkyl chains (less polar monomers), indicating lower compatibility with the polar solvent mixture.[26] While smaller particles were produced with these less polar monomers, it was found that $d_{p}$ formed by poly(2-ethylhexyl methacrylate) and poly(lauryl methacrylate) was less sensitive to the change of medium polarity (modified by increasing the methanol amount in the binary solvent) compared to the particles synthesized with shorter chain alkyl methacrylates. The solvency of the reaction medium relative to that of the polymer being formed plays an important role in the polymerization, as it affects the critical chain length of the polymerizing chains; thus it influences particle size in heterogeneous system.[27] Lok and Ober[28] discussed dispersion polymerization of ST with the steric stabilizer hydroxypropyl cellulose in methyl cellosolve (MeCell)/ethanol and illustrated that monodispersed and larger particles were produced with an increased MeCell fraction in the solvent mixure, due to the better affinity 
between the core polymer and the medium. Hansen solubility parameters have also been applied to predict the partition behavior of cytotoxic solutes between polymer phase and aqueous phase.[29]

The aforementioned research studies were done in batch systems, in which the presence of the monomer at the start of the reaction influences the polarity of the continuous phase. In the semibatch system, however, monomer concentration in the system is low and thus its presence can be neglected when considering the solvency of the continuous phase. Hansen solubility parameters for the mixed solvents, copolymer dispersants, and copolymer cores reported in Table 3 were calculated via the root-mean-square volume weighted average of the pure component values estimated through HSPiP 5.0.03 software (see Supplementary Data). The overall Hansen solubility parameter $\left(\delta, \mathrm{HSP}, \mathrm{MPa}^{1 / 2}\right)$ is composed of three parts, where $\delta_{\mathrm{d}}, \delta_{\mathrm{p}}$ and $\delta_{\mathrm{h}}$ are the dispersion forces, polar forces and hydrogen-bonding effects, respectively, as shown in Equation 1.[29,30]

$$
\delta^{2}=\delta_{d}^{2}+\delta_{p}^{2}+\delta_{h}^{2}
$$

The values in Table 3 indicate that the HSP for the core polymer decreases slightly with the addition of non-polar ST, and increases with the addition of the functional monomers (GMA, MAA and HEA). These small changes in core polymer polarity do not correlate with the changes in particle size observed experimentally (Figure 4), suggesting that the reactivity differences of the monomers (and their interaction with the reactive macromer dispersant) are more important in controlling nucleation and final particle size. However, HSP values for the dispersant increase as the fraction of HEMA is increased, moving away from the value for heptane/xylene mixture and closer towards the values of the core polymer. This lowered affinity between dispersant and the continuous phase is consistent with the poorer performance of $\mathrm{BH}_{10}$ and $\mathrm{BH}_{15}$ used as stabilizer in the NAD system. The result suggests that stability might be regained by increasing the polarity of the solvent mixture or decreasing the polarity of the hydroxyl-containing macromer. These strategies will be experimentally investigated as a means to produce stable NAD systems with increased hydroxyl functionality in the macromer dispersant. 


\section{Conclusions}

A series of non-aqueous dispersions were made with core compositions varied through the introduction of ST and functional monomers GMA, MAA, and HEA using both BMA homopolymer and BMA/HEMA macromer (5 mol\% HEMA) dispersants. NADs made with the MA homopolymer core have the smallest particle size and highest fraction of incorporated dispersant. Introducing crosslinker $(5.8 \mathrm{wt} \%)$ and HEA $(10 \mathrm{wt} \%)$ to the core recipe resulted in slightly larger $d_{p}$ and no change in the level of incorporated dispersant. The addition of $10 \mathrm{wt} \%$ ST had a larger impact compared with the functional monomers due to its slower polymerization rate and/or the formation of sterically hindered radicals through reaction with the macromers. Despite these small variations, the dispersions produced were all stable, with high solids content $(60 \mathrm{wt} \%)$ and small particle size $(<180 \mathrm{~nm})$, features desired for potential commercial application of macromer dispersants. However, the NAD systems were not stable when produced with BMA/HEMA macromer containing higher hydroxyl levels (10 and 15 mol\%). This loss of stability was qualitatively interpreted using Hansen solubility parameters, which seems promising as a tool to tune solvent and dispersant choices to produce stable NADs tailored to fit product application needs. Further work in this direction is underway.

Acknowledgement. The authors acknowledge the valuable advice and suggestions from Dr. Jeffery W. Johnson and Dr. Andrew P. Stamegna of Axalta Coating Systems. This work was financially supported by Axalta Coating Systems (Wilmington, U.S.A) and the Natural Sciences and Engineering Research Council of Canada.

\section{References}

[1] R. J. Barsotti, L. A. Lewin, C. Scopazzi, Coating Compositions Containing Non-Aqueous Dispersion Polymers Having a High Glass Transition Temperature, 1998.

[2] K. E. J. Barrett, Dispersion Polymerisation in Organic Media, Br. Polym. J. 5 (1973) 259271.

[3] A. J. Paine, Dispersion Polymerization of Styrene in Polar Solvents. 7. A Simple Mechanistic Model to Predict Particle Size, Macromolecules. 23 (1990) 3109-3117. 
[4] F. L. Baines, S. Dionisio, N. C. Billingham, S. P. Armes, Use of Block Copolymer Stabilizers for the Dispersion Polymerization of Styrene in Alcoholic Media, Macromolecules. 29 (1996) 3096-3102.

[5] R. Dowbenko, D. P. Hart, Nonaqueous Dispersions as Vehicles for Polymer Coatings, Ind. Eng. Chem. Prod. Res. Dev. 12 (1973) 14-28.

[6] S. Kobayashi, H. Uyama, J. H. Choi, Y. Matsumoto, Preparation of Micron-Size Monodisperse Poly(methyl methacrylate) Particles Using Poly(2-oxazoline) Macromonomer, Polym. Int. 30 (1993) 265-270.

[7] J. S. Shay, R. J. English, R. J. Spontak, C. M. Balik, S. A. Khan, Dispersion Polymerization of Polystyrene Latex Stabilized with Novel Grafted Poly(ethylene glycol) Macromers in 1-Propanol/Water, Macromolecules. 33 (2000) 6664-6671.

[8] S. M. Klein, V. N. Manoharan, D. J. Pine, F. F. Lange, Preparation of Monodisperse PMMA Microspheres in Nonpolar Solvents by Dispersion Polymerization with a Macromonomeric Stabilizer, Colloid Polym. Sci. 282 (2003) 7-13.

[9] W. Yang, R. A. Hutchinson, Investigating the Effectiveness of Reactive Dispersants in Non-Aqueous Dispersion Polymerization, Macromol. React. Eng. 10 (2016) 71-81.

[10] M. Ali Parsa, I. Kozhan, M. Wulkow, R. A. Hutchinson, Modeling of Functional Group Distribution in Copolymerization: A Comparison of Deterministic and Stochastic Approaches, Macromol. Theory Simulations. 23 (2014) 207-217.

[11] P. Cacioli, D. G. Hawthorne, R. L. Laslett, E. Rizzardo, D. H. Solomon, Copolymerization of $\omega$-Unsaturated Oligo(Methyl Methacrylate): New Macromonomers, J. Macromol. Sci. Chem. 23 (1986) 839-852.

[12] S. Kiatkamjornwong, C. Kongsupapsiri, Control of Monodisperse Particle Size of Styrenic-acrylate Copolymers in Dispersion Copolymerization, Polym. Int. 49 (2000) $1395-1408$.

[13] W. Yang, D. Yang, J. Hu, C. Wang, S. Fu, Dispersion Copolymerization of Styrene and Other Vinyl Monomers in Polar Solvents, J. Polym. Sci. Part A Polym. Chem. 39 (2001) $555-561$. 
[14] J. Song, L. Chagal, M. A. Winnik, Monodisperse Micrometer-Size CarboxylFunctionalized Polystyrene Particles Obtained by Two-Stage Dispersion Polymerization, Macromolecules. 39 (2006) 5729-5737.

[15] J. Song, F. Tronc, M. A. Winnik, Monodisperse, Controlled Micron-size Dye-labeled Polystyrene Particles by Two-Stage Dispersion Polymerization, Polymer. 47 (2006) 817825.

[16] S. Jiang, E. D. Sudol, V. L. Dimonie, M. S. El-Aasser, Dispersion Copolymerization of Methyl Methacrylate and n-Butyl Acrylate, J. Polym. Sci. Part A Polym. Chem. 45 (2007) 2105-2112.

[17] A. N. Nikitin, M. C. Grady, G. A. Kalfas, R. A. Hutchinson, Investigation of Catalytic Chain Transfer Copolymerization of Methacrylates, Macromol. React. Eng. 2 (2008) 422435.

[18] A. C. Simmons, The Refractive Index and Lorentz-Lorenz Functions of Propane, Nitrogen and Carbon-dioxide in the Spectral Range 15803-22002 $\mathrm{cm}^{-1}$ and at $944 \mathrm{~cm}^{-1}$, Opt. Commun. 25 (1978) 211-214.

[19] L. Grunberg, A. H. Nissan, Mixture Law for Viscosity, Nature. 164 (1949) 799-800.

[20] R. A. Cockburn, T. F. L. McKenna, R. A. Hutchinson, A Study of Particle Nucleation in Dispersion Copolymerization of Methyl Methacrylate, Macromol. React. Eng. 5 (2011) 404-417.

[21] K. E. J. Barrett, H. R. Thomas, Kinetics of Dispersion Polymerization of Soluble Monomers. I. Methyl Methacrylate, J. Polym. Sci. Part A-1 Polym. Chem. 7 (1969) 26212650 .

[22] S. Hamzehlou, Y. Reyes, R. Hutchinson, J. R. Leiza, Copolymerization of n-Butyl Acrylate and Styrene: Terminal vs Penultimate Model, Macromol. Chem. Phys. 215 (2014) $1668-1678$.

[23] F. H. Walker, Introduction to Polymer Resins, Federation of Societies for Coatings Technology, 1999: pp. 32. 
[24] K. Adamsons, G. Blackman, B. Gregorovich, L. Lin, R. Matheson, Oligomers in the Evolution of Automotive Clearcoats: Mechanical Performance Testing as a Function of Exposure, Prog. Org. Coatings. 34 (1998) 64-74.

[25] W. Yang, Non-Aqueous Dispersion Polymerization for Automotive Coating Applications, Queen's University, 2016.

[26] Y. Saadat, S. Hosseinzadeh, F. Afshar-Taromi, H. Eslami, S. Abdolbaghi, Generalizing the Polymerization Conditions for the Production of Monodisperse Polymeric Particles via Dispersion Polymerization, Colloid Polym. Sci. 291 (2013) 937-944.

[27] D. Horák, F. Švec, J. M. J. Fréchet, Preparation and Control of Surface Properties of Monodisperse Micrometer Size Beads by Dispersion Copolymerization of Styrene and Butyl Methacrylate in Polar Media, J. Polym. Sci. Part A Polym. Chem. 33 (1995) 23292338.

[28] K. P. Lok, C. K. Ober, Particle Size Control in Dispersion Polymerization of Polystyrene, Can. J. Chem. 63 (1985) 209-216.

[29] S. L. Bacon, E. C. Peterson, A. J. Daugulis, J. S. Parent, Selecting Polymers for TwoPhase Partitioning Bioreactors (TPPBs): Consideration of Thermodynamic Affinity, Crystallinity, and Glass Transition Temperature, Biotechnol. Prog. 31 (2015) 1500-1507.

[30] C. M. Hansen, Hansen Solubility Parameters A User's Handbook, Taylor \& Francis Group, 2007. 


\section{List of Figures}

Figure 1. MMDs of Dispersant M1 (solid line), a dispersion sample taken 60 min after the start of polymerization (dotted line), and the final dispersion product (dashed line) from recipe AS

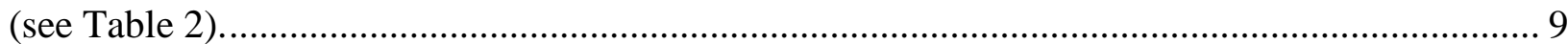

Figure 2. Weight-average molar mass of MA homopolymer (filled bars, sample named as "A" in Table 2) and MA/ST copolymer (unfilled bars, sample named as "AS" in Table 2) NADs prepared using two different BMA macromers. 10

Figure 3. The mass fraction of incorporated dispersant $\left(w_{\text {inc }}\right)$ measured for NADs made with two macromer dispersants M1 (filled bars) and M2 (unfilled bars) and various core compositions (see Table 2) 11

Figure 4. Average particle size measured for NADs made with two macromer dispersants M1 (filled bars) and M2 (unfilled bars) and various core compositions (see Table 2).

Figure 5. Non-aqueous dispersions of MA homopolymer core prepared with two different HEMA-containing macromers: (a) stable NAD produced with $\mathrm{BH}_{5}$ (5 mol\% HEMA); (b) unstable NAD produced with $\mathrm{BH}_{10}(10$ mol\% HEMA).

Figure 6. MMDs of macromer dispersants and $\mathrm{NADs} \mathrm{BH}_{0}$ (solid line), $\mathrm{BH}_{5}$ (dashed line), NAD $\mathrm{A}-\mathrm{BH}_{0}$ (dotted line) and $\mathrm{NAD} \mathrm{A}-\mathrm{BH}_{5}$ (dashed-dotted line). 15

Figure 7. A comparison of the dispersant MMDs (solid lines) with the soluble polymer recovered from the final non-aqueous dispersion by heptane treatment (dashed lines): (a) $\mathrm{BH}_{0}$ and NAD A$\mathrm{BH}_{0}$; (b) $\mathrm{BH}_{5}$ and NAD A-BH 5 ; (c) $\mathrm{BH}_{5}, \mathrm{NAD} A H S X-\mathrm{BH}_{5}$. In (c), the MMD of the soluble polymer exacted by $80 / 20$ heptane/xylene mixture is also shown (dotted line). 
Table 1. Macromer dispersants utilized for the preparation of NADs.

\begin{tabular}{cccc}
\hline Macromer & $\begin{array}{c}\text { Composition } \\
(\mathrm{BMA} / \mathrm{HEMA})\end{array}$ & $\mathrm{M}_{\mathrm{n}}(\mathrm{Da})^{\mathrm{a})}$ & $\mathrm{M}_{\mathrm{w}}(\mathrm{Da})^{\mathrm{a})}$ \\
\hline $\mathrm{M} 1$ & $100 / 0$ & 5600 & 9200 \\
$\mathrm{M} 2$ & $100 / 0$ & 8300 & 15000 \\
$\mathrm{BH}_{0}^{\mathrm{b})}$ & $100 / 0$ & 7100 & 12000 \\
$\mathrm{BH}_{5}$ & $95 / 5$ & 7800 & 14200 \\
$\mathrm{BH}_{10}$ & $90 / 10$ & 6500 & 11800 \\
$\mathrm{BH}_{15}$ & $85 / 15$ & 5400 & 9200 \\
\hline
\end{tabular}
a) MW values are reported according to polystyrene calibration.
b) This sample represents a BMA homopolymer macromer, without the presence of HEMA units.

Table 2. Core compositions explored for NADs prepared with macromer dispersants. ${ }^{\mathrm{a}}$

\begin{tabular}{cccccc}
\hline Sample & MA & GMA & MAA & ST & HEA \\
\hline A & 100 & - & - & - & - \\
AS & 90 & - & - & 10 & - \\
AX & 94.2 & 1.6 & 4.2 & - & - \\
AHX & 84.2 & 1.6 & 4.2 & - & 10 \\
AHSX & 74.2 & 1.6 & 4.2 & 10 & 10 \\
\hline
\end{tabular}

a) The values are based on the fractions of each monomer in the core as $\mathrm{wt} \%$. 
Table 3. Hansen solubility parameters $\left(\mathrm{MPa}^{1 / 2}\right)$ calculated for solvent mixtures, macromer dispersants, and core compositions.

\begin{tabular}{|c|c|c|c|c|c|}
\hline & Sample $^{a)}$ & $\delta_{\mathrm{d}}$ & $\delta_{\mathrm{p}}$ & $\delta_{\mathrm{h}}$ & $\delta$ \\
\hline Solvent Mixture & heptane/xylene & 16.2 & 0.4 & 1.1 & 16.23 \\
\hline \multirow{4}{*}{ Macromer } & $\mathrm{BH}_{0}^{\mathrm{b})}$ & 16.1 & 1.4 & 3.5 & 16.5 \\
\hline & $\mathrm{BH}_{5}$ & 16.15 & 1.58 & 3.93 & 16.7 \\
\hline & $\mathrm{BH}_{10}$ & 16.2 & 1.76 & 4.36 & 16.9 \\
\hline & $\mathrm{BH}_{15}$ & 16.25 & 1.94 & 4.79 & 17.1 \\
\hline \multirow{5}{*}{ Core Polymer } & $\mathrm{A}$ & 17.4 & 4.3 & 6.5 & 19.1 \\
\hline & $\mathrm{AS}$ & 17.5 & 3.9 & 6.1 & 19.0 \\
\hline & $\mathrm{AX}$ & 17.5 & 4.3 & 6.8 & 19.2 \\
\hline & AHX & 17.4 & 4.7 & 7.7 & 19.6 \\
\hline & AHSX & 17.6 & 4.3 & 7.4 & 19.6 \\
\hline
\end{tabular}

a) See Tables 1 and 2 for details; b) PBMA homopolymer. 
Supplementary Data
Click here to downlo:

Click here to download Supplementary Data: NAD - Weiwei 2nd manuscript_SI.pdf 